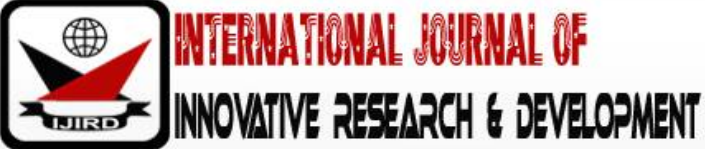

ISSN 2278 - 0211 (Online)

\section{Effect of Temperature on Acrylamide in Potato (Solanum Tuberosum) Chips}

\author{
Obed Mainya Nyabaro \\ Lecturer, Department of chemistry, Kisii University, KISII, Kenya \\ Dr. Tabitha Wanjau \\ Lecturer, Department of Health sciences, Kisii University, KISII, Kenya \\ Lusweti Kituyi \\ Lecturer, Department of chemistry, University of Eldoret, Eldoret, Kenya
}

\begin{abstract}
:
In the recent past scientists in Britain, Sweden and South Africa have discovered that the process of cooking starchy foods under high temperatures produces acrylamide in those foods. Fried products including potato chips and crisps have been reported to contain high levels of acrylamide, a potential carcinogenic and neurotoxic substance of great concern to consumers. Samples were purposefully obtained from some retail outlets in Kisii County in the most visited hotels and retail centers. The effect of temperature was analysed using Time-temperature data which were acquired after every second using a digital multimeter and later analytically prepared before analysis to avoid contamination and also to ease the analytical procedure before they were injected to the High Performance Liquid Chromatography for analysis. Both descriptive and inferential statistical tests including analysis of variance (ANOVA) and least significant difference test (LSD) for the variables were carried out using the Statistical Analysis System (SAS) version 9.1.3.
\end{abstract}

Keywords: Toxicology, acrylamide, carcinogen, neurotoxicant, chromatography

\section{Introduction}

As the amount of unintended hazardous substances is increased during food production, processing, and cooking, researcher's interest towards these substances is increasing. Heterocyclic amines (HCAs) and acrylamide could be presented as the unintended harmful substances in cooked foodstuffs [3], by the Maillard reaction. HCAs are known as mutant substances, which emerge when heating (fried, broiled, or barbecued) foodstuffs rich in amino acids or creatine. They are categorized into the amino carboline group or amino-imidazo-azaarene (AIA) group according to their chemical structures [13]. This results from cooking of starchy foods under certain temperatures. Starchy foods are harmful to the human health; in the recent past scientists in Britain have discovered that the process of cooking starchy foods such as chips, potato chips, and bread at high temperatures $(120 \mathrm{oC})$ leads to the production of acrylamide in the heating process [11].

It has been established by various studies that acrylamide is formed in carbohydrate-rich foods that undergo high temperature processing such as conventional fried potatoes and not

Boiling [2; 9; 28]. Despite the claim by [27] that the Swedish report triggered a worldwide interest, developing countries seem to have been ignorant of the implications of acrylamide in food. There are no data on occurrence of acrylamide pertinent to countries and regions where the types of foods, methods of food preparation are different from those in Western nations.

Potato chips as foodstuff are usually consumed in three major forms; fried chips, fried crisps or just boiled. Crisps are fragile but firm slices that have been processed through deep frying and edible salt or acceptable food grade spices colour and flavour may have been added [11]. On the other hand, Potato chips which are locally referred to as chips are long; thinly cut slices of which have been deep fried [12;4].

Potato chips and crisps are among the starchy foods commonly used as a tuberous crop from the perennial night shade Solanum tuberosum. They have a high content of carbohydrates and vitamin $\mathrm{C}$ and thus, forming an important component of a balanced diet. Worldwide, it is the fourth most widely grown food crop after rice, wheat and maize [8]. In Kenya, potato is the second most valuable cash and food crop after the cereal grains [6].

Acrylamide doesn't appear to be in raw foods themselves. It is formed when certain starchy foods that are cooked at high temperatures causing a chemical reaction between sugars and an amino acid (asparagines) in the foods which forms acrylamide. Longer cooking times and cooking at higher temperatures on starchy foods increases the amount of acrylamide [1].

Acrylamide doesn't appear to be in raw foods because it is formed upon cooking of starchy foods at high temperatures beyond 1200C where chemical reactions between certain sugars and amino acids in the food form 
acrylamide. Acrylamide is a chemical compound with the chemical formulaC3H5NO and an average mass of $71.08 \mathrm{~g} / \mathrm{mol}$. Its IUPAC name is prop-2-enamide. It is a highly water soluble vinyl monomer formed from the hydration of acrylonitrile. It is primarily used in research laboratories for electrophoresis, chromatography, and electron microscopy and in the sewage and wastewater treatment industries [15]. It decomposes non-thermally to form ammonia, and thermal decomposition produces carbon monoxide, carbon dioxide, and oxides of nitrogen[14]. The figure below shows the structure of acrylamide.

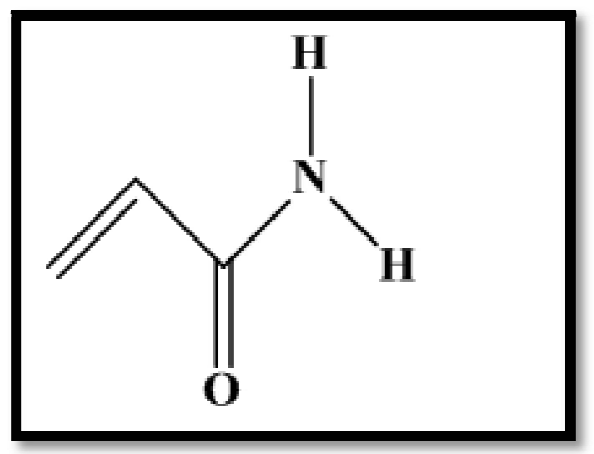

Figure 1: Structure of Acrylamide

\section{Methodology}

\subsection{Apparatus, Chemicals and Solvents}

All solvents and chemicals used in the analysis procedure were of analytical grade. They were purchased from Merck (Darmstadt, Germany) agents in Kenya. Water was double distilled and further purified using a water purification system since the instruments used were highly sensitive and to avoid breakdown after calibration for the HPLC instrument, analytical grade of HPLC water was used.

Acrylamide (standard) (99\%) and 2H3 -labeled acrylamide (isotopic purity 98\%) was purchased from SigmaAldrich (St. Louis, MO) and Cambridge Isotope Laboratories (Andover, MA), respectively. Methanol (HPLC-grade) was supplied by Merck (Darmstadt, Germany). Water was purified with a Milli-Q system (Millipore, Bedford).

AccuBOND Si (6 mL, $500 \mathrm{mg}$ ) solid-phase extraction (SPE) cartridges were supplied by Agilent Technologies (Santa Clara, $\mathrm{CA})$. Standards and reagents stock solution of acrylamide $(1 \mathrm{mg} / \mathrm{mL})$ and $2 \mathrm{H} 3$-labeled acrylamide $(0.1 \mathrm{mg} / \mathrm{mL})$ were prepared by dissolving suitable amount of the compounds in water. These solutions were then appropriately diluted with water to prepare working standards at 10 and $4 \mu \mathrm{g} / \mathrm{mL}$, respectively. All stock solutions and working standards were kept at $4^{\circ} \mathrm{C}$ for a month.

\subsection{Sample Preparation and Analysis}

Despite acrylamide being a relatively new contaminant for food analysts, intensive method development and refinement have been carried out. Various methods of determining the concentration of acrylamide in foods have been addressed. Among all these methods, three have been widely used for extraction and quantification of acrylamide in foods. The samples were prepared for the analysis of potato chips and crisps by weighing $1 \mathrm{~g}$ of crushed potato chips and mixing with $10 \mathrm{~mL}$ methanol on a wrist action shaker for 20 minutes to enable the sample to fully soak in the extract solution. The samples were refrigerated for 2 minutes for easier extraction of the oily top layer to avoid interferences. The supernatant was filtered through a $0.45 \mu \mathrm{m}$ nylon syringe filter and the filtrate discarded and the residue stored for further cleanup and analysis.

The residue was conditioned through a carboPrep ${ }^{\mathrm{TM}} 200 \mathrm{SPE}$ tube using, $6 \mathrm{~mL}$ of the sample in $2 \mathrm{~mL}$ acetone and 2 $\mathrm{mL}$ methanol. The sample solution was allowed to pass through the tube by gravity and 0.5-1.0 mL water was run through the tube to wash the sample. A vacuum pump was used to dry excess water from tube for 1 minute and eluted with $2 \mathrm{~mL}$ of acetone through gravity therefore ready for analysis in the instrument. Many sample extracts can be analysed directly, however, sample cleanup and solvent pre-concentration was essential.

Detection and quantitation of acrylamide in chips and crisps were determined using High performance liquid chromatography (HPLC). Agilent 1100 (Waldbronn, Germany) HPLC system consisting of a quaternary pump with vacuum degasser, a DAD was used. Chromatographic separations were performed on an ODS-3 C18 column (250 mm $\times 4.6 \mathrm{~mm}$, Intersil, Japan).

\subsection{Determination of Effect of Temperature}

This test was done to determine the optimum temperature at which acrylamide formation starts in the frying process of potatoes. Fresh potato samples were purchased from the market, washed and peeled by hand and sliced into uniform thickness about $2 \mathrm{~mm}$ by pro-v-slicer which has adjustable and reversible stainless steel sliding blades (model 1668, Taiwan). Then slices were soaked in water to remove their surface starches, dried using special paper towel and finally weighed by digital balance with $0.0001 \mathrm{~g}$ precision.

A stainless steel pan with 6 litre capacity, $24.3 \mathrm{~cm}$ inner diameter and $0.5 \mathrm{~cm}$ thickness was used for frying purposes. This pan had a stainless steel lid with hermetic property, which thermometer, gauge pressure, food transporting 
rod, vacuum and gas valves are equipped on. An electrical heater (model thermal, HK3-2, Germany) was used as a heat source. This heater had an automatic time-temperature control with digital thermometer.

A $10 \mathrm{~g}$ of the sample were heated in oil up to a temperature of $100 \mathrm{oC}$ and removed then analyzed for the quantity of acrylamide. Different sets of $10 \mathrm{~g}$ samples were heated to temperatures ranging between $110 \mathrm{oC}$ to $200 \mathrm{oC}$. All these samples were analyzed to determine the quantity of acrylamide and thus the optimum temperature at which acrylamide formation started; the effect of temperature on colour and moisture content. To ensure the temperature aspect was fully adhered to this was repeated to a certain number of replicates in different temperatures that were studied to ascertain accuracy and precision of the results that were obtained.

\section{Results}

\subsection{Temperature - Acrylamide Relationship}

To determine the temperature at which acrylamide formation starts and how it increases in relation to temperature increase, potato slices samples were fried at $100 \mathrm{oC}, 105 \mathrm{oC}, 110 \mathrm{o} C, 115 \mathrm{oC}, 120 \mathrm{oC}, 125 \mathrm{oC}, 130 \mathrm{oC}, 135 \mathrm{oC}$, $140 \mathrm{oC}, 160 \mathrm{oC}, 180 \mathrm{oC}$, then finally $200 \mathrm{oC}$. All these samples were analyzed to determine the quantity of acrylamide and thus the optimum temperature at which acrylamide formation starts.

\begin{tabular}{|c|c|c|}
\hline Sample Id & Temp & CONC. (mg/ kg) \\
\hline CT1 & $100^{\circ} \mathrm{C}$ & 0.1011 \\
\hline CT2 & $105^{\circ} \mathrm{C}$ & 0.2095 \\
\hline CT3 & $110^{\circ} \mathrm{C}$ & 0.6360 \\
\hline CT4 & $105^{\circ} \mathrm{C}$ & 1.0073 \\
\hline CT5 & $110^{\circ} \mathrm{C}$ & 1.0897 \\
\hline CT6 & $115^{\circ} \mathrm{C}$ & 1.1900 \\
\hline C2 & $120^{\circ} \mathrm{C}$ & 1.5357 \\
\hline CT7 & $125^{\circ} \mathrm{C}$ & 1.6398 \\
\hline CT8 & $130^{\circ} \mathrm{C}$ & 1.8910 \\
\hline CT9 & $135^{\circ} \mathrm{C}$ & 2.1596 \\
\hline CT10 & $140^{\circ} \mathrm{C}$ & 2.2179 \\
\hline C11 & $160^{\circ} \mathrm{C}$ & 3.6514 \\
\hline C5 & $180^{\circ} \mathrm{C}$ & 4.6434 \\
\hline CK7 & $200^{\circ} \mathrm{C}$ & 5.4476 \\
\hline
\end{tabular}

Table 1: Temperature - Acrylamide Relationship

The Figure 2 presents a graph showing the increase in the concentration of acrylamide in the potato chips as the temperature increases.

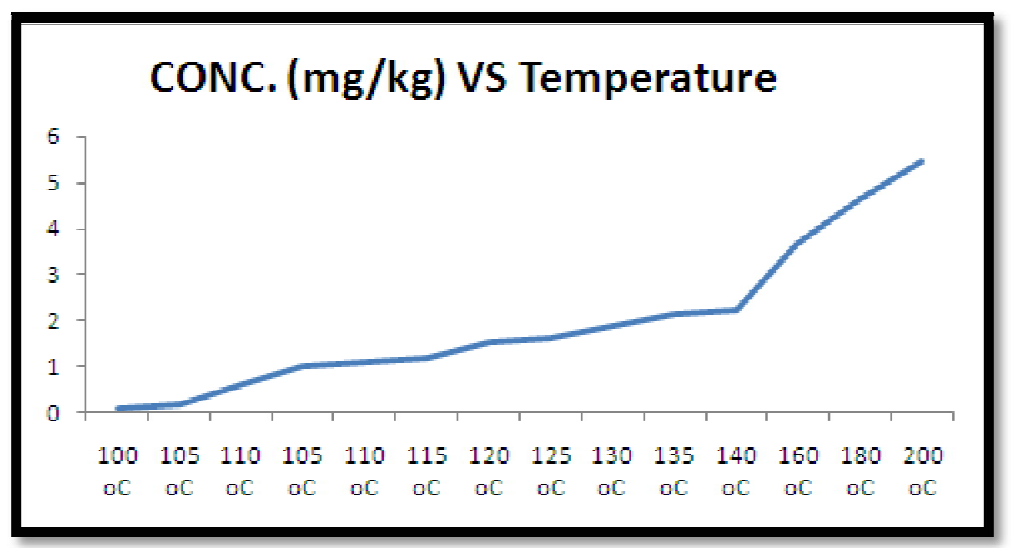

Figure 2: Acrylamide - Temperature Relationship

The results above show that the content of acrylamide increased with increasing temperature. Fig. 3, it can be seen that the increase in acrylamide concentration with increasing frying temperature followed an exponential function from $1100 \mathrm{C}$ up to 2000C. Since acrylamide formation is also dependent on initial sugar and asparagine contents[16]. Acrylamide formation started at $1100 \mathrm{C}$ with the initiation of milliard reaction at this temperature. The conversion of sugar and asparagine in the potato slices increases as temperature increases up to 1900C then the conversion ends with the exhaustion of the sugars in the sample. These results agree with those of Matthäus [20]who showed that the amount of acrylamide was relatively low at temperatures between 150 and 1750C, whereas a drastic increase was observed at higher temperatures. The amount of acrylamide formed in potato chips was about $0.80 \mathrm{mg} / \mathrm{kg}$ at $1700 \mathrm{C}$ and increased to about $3.70 \mathrm{mg} / \mathrm{kg}$ at 1900C. Gertz \& Klostermann, [5]showed that the formation of acrylamide was accelerated above 1750C but stagnated at 1900C. Pedreschi [23]also reported a significant increase in acrylamide level as the frying temperature increased from 1500C to 1900C. They found that the acrylamide content of potato slices was about $0.50 \mathrm{mg} / \mathrm{kg}$ at $1500 \mathrm{C}$ as 
opposed to about $4.500 \mathrm{mg} / \mathrm{kg}$ at 1900C. The process of conversion of asparagine to acrylamide through Maillard reaction is shown.

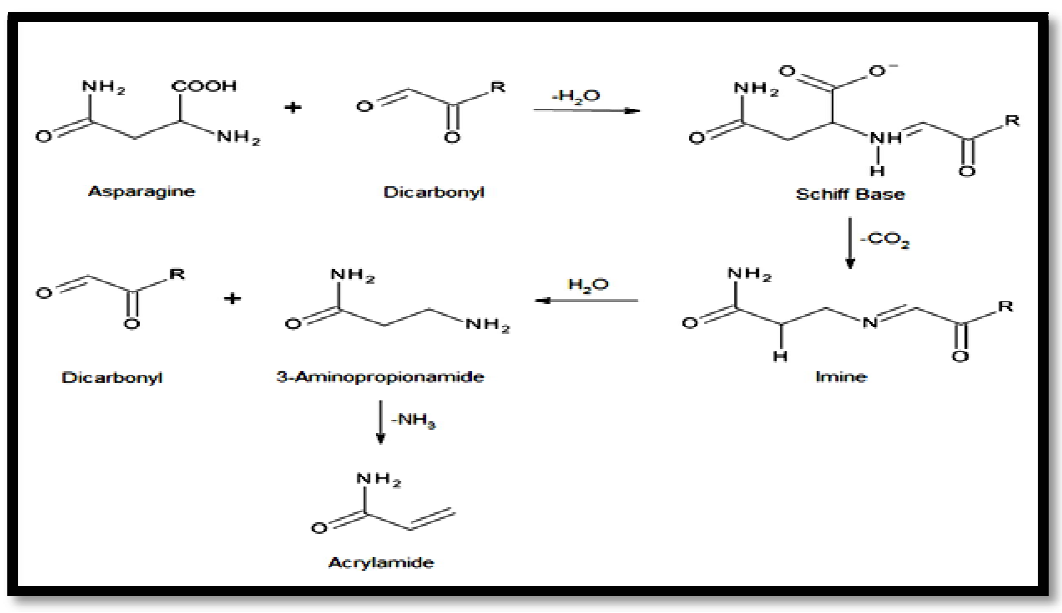

Figure 3: Mechanism of Conversion of Asparagine to Acrylamide through Maillard Reaction

The reaction is a form of non-enzymatic browning which typically proceeds rapidly from around 140 to $165{ }^{\circ} \mathrm{C}$ ( 2840 to $329^{\circ} \mathrm{F}$ ). The reactive carbonyl group of the sugar reacts with the nucleophilic amino group of the amino acid, and forms a complex mixture of poorly characterized molecules responsible for a range of odours and flavours [26]. This process is accelerated in an alkaline environment as the amino groups (RNH3+) are deprotonated and, hence, have an increased nucleophilicity [25]. The type of the amino acid determines the resulting flavour. This reaction is the basis of the flavouring industry. At high temperatures, acrylamide can be formed.

Acrylamide was shown to form even at temperatures below 1200C when the moisture content becomes sufficiently low upon prolonged frying. The core temperature does not exceed 103-104 C during frying for durations commonly applied in home and restaurant French chips preparation practices [24].

From the results, it is evident that temperature is the main cause of increment in acrylamide levels since as the temperature increases the acrylamide levels begin to rise. Raw potatoes do not contain acrylamide, but high amounts of the precursors for its formation [18]; [24]. Fried potatoes vary in the amounts of acrylamide formed upon exposure to a given thermal treatment [17]. The resulting acrylamide concentration depends on the concentrations of the precursors, as well as the processing conditions. The fact that acrylamide is not formed during boiling indicates that higher temperatures and/ or low moisture conditions are needed for its formation. During heating at atmospheric conditions, higher temperatures can be reached only if simultaneous drying takes place, which is the case in frying [24].

Temperature and time have been shown to be significant factors affecting the amount of acrylamide formed in potatoes during frying [20]; [23]. However, the temperature of frying oil was reported instead of the change of local internal temperatures during frying. Since a temperature distribution in the product occurs due to conductive resistance to heat transfer and evaporation of water from the product, the rate of acrylamide formation will not be the same for layers from surface to core of product [24].

In the present research investigation, it is evident that temperature is a core issue in formation of acrylamide, it can further be seen that for a constant frying time the increase in acrylamide concentration with increasing frying temperature followed an exponential function where at a constant frying temperature the graph of concentration verses temperature was linear with a greater slope at higher temperatures. Similar results have been reported previously by other researchers such as Vural [7] found out that Frying time had little or no effect on the formation of acrylamide in potato strip at $150 \mathrm{o} \mathrm{C}$ while increasing oil temperature resulted in higher acrylamide levels after the same frying time. Acrylamide content after frying at $190 \mathrm{o}$ C for 3 min was about 40 times larger than that after 9 min of frying at $150 \mathrm{o} C$ this is in line with the current research.

Gökmen [7]got similar results in their study of the relationship between the acrylamide formation and timetemperature history of surface and core regions of potato chips. Their findings showed that acrylamide formation started at $1200 \mathrm{C}$ and increased exponentially with increase in temperature. Acrylamide content of the surface was found to be 72 , 2747 and $6476 \mathrm{mg} / \mathrm{g}$ after frying for $9 \mathrm{~min}$ at 1500C, 1700C and 1900C, respectively. The core was free of acrylamide after frying for $9 \mathrm{~min}$ at $1500 \mathrm{C}$ and $1700 \mathrm{C}$, while only $376 \mu \mathrm{g} / \mathrm{g}$ of acrylamide was formed at 1900C.

Results as per vural [24] indicate a constant increase in acrylamide levels followed an exponential function similar to the results in this study where at a constant frying temperature the graph was linear with a greater slope at higher temperatures

The findings also correlate with those of Pedreschi [22] who found out that acrylamide formation starts at 1500C and increased exponentially with increasing temperature and moisture. However, they discovered that acrylamide formation can be reduced by pre-soaking the potato chips in water or citric acid before frying. Soaked slices showed on average a reduction of acrylamide formation of $27 \%, 38 \%$ and $20 \%$ at 1500C, 1700C and 1900C, respectively. Soaking reduces the concentrations of glucose and asparagine content in the potato chips before frying. 
Ogolla [21] found that acrylamide formation begins at $120^{\circ} \mathrm{C}$ and optimal development occurs between $160^{\circ} \mathrm{C}$ and $180^{\circ} \mathrm{C}$. The use of low frying temperature (under $160^{\circ} \mathrm{C}$ ) reduces the concentration of acrylamide.

\section{Conclusion}

This research addressed issues that are specific to acrylamide presence and control in order to guarantee production of safe and wholesome potato products. Advice farmers to harvest fully mature potatoes since the pre mature ones have a high sugar levels than the mature potatoes therefore harvesting should be at the maturity time.

It is evident that cooking potato chips at high temperatures leads to the rise in acrylamide levels therefore potato chips should be cooked at low temperatures to avoid increase in acrylamide levels. Good Agricultural Practices (GAPs) and Good Manufacturing Practices (GMPs) is important to help control acrylamide levels associated with all stages of production, handling and processing of potato products.

Consumers and processors should be provided with the recommended practices that can be undertaken to reduce the level of formation of acrylamide in potato products. Specific emphasis is placed on the quality of raw materials and processing conditions, control of other ingredients and food processing.

\section{References}

i. American Cancer Society (2016) Medical and Editorial Content March.

ii. Carrieri G., Anese M., Quarta B., De Bonis M.V., Ruocco G. (2010). Evaluation of acrylamide formation in potatoes during deep-frying: The effect of operation and configuration.J. Food Eng., 98(2): 141- 149.

iii. Felton, J.S., Malfatti, M.A., Knize, M.G., Salmon, C.P., Hopmans, E.C. and Wu, R.W. (1997) Health risks of heterocyclic amines. Mutat. Res., 376, 37-41.

iv. Franco Pedreschi, Kit Granby and Jørgen Risum (2010). Acrylamide Mitigation in Potato Chips by Using NaCl.

v. Gertz, C., Klostermann, S. (2002). Analysis of acrylamide and mechanisms of its formation in deep-fried products. Eur.J. Lipid Sci.Technol. 101; 762-771.

vi. Gitau, M. (2013). Small scale growing business in Kenya Youth Agro Environment Initiative. Retrieved June 23, 2014, from http:/ / yagrein.blogspot.com/ 2012/ 05/ small-scale-es-growing-business.html

vii. Gökmen, V., Palazoğlu, T. K., \& Şenyuva, H. Z. (2006). Relation between the acrylamide formation and timetemperature history of surface and core regions of French fries. Journal of Food Engineering, 77(4), 972-976.

viii. Hijmans, R.J. and Spooner, D.M. (2001). Geographic distribution of wild species. American Journal of Botany 88 (11): 2101-12.

ix. Michalak J., Gujska E., Klepacka J. (2011). The Effect of Domestic Preparation of Some Potato Products on Acrylamide Content. Plant foods for human nutrition (Dordrecht, Netherlands), pp. 307-312.

x. Pedreschi, F., Moyano, P., Kaack, K., and Granby, K. (2005). Colour changes and acrylamide formation in fried potato slices. Food Research International, 38(1), 1-9.

xi. Sivasankar, B. (2002). Food Processing and Preservation. PHI Learning Pvt. Ltd. pp. 175-177.ISBN 8120320867

xii. Schehr, L. R. and Weiss, A. S. (2001). Potato Food: On the Table on the Page and in Potato Culture. Abingdon: Routledge. p. 158.ISBN 0415936284.

xiii. Skog, K.I., Johansson, M.A. and Jagerstad, M.I. (1998) Carcinogenic heterocyclic amines in model systems and cooked foods: a review on formation, occurrence and intake. Food Chem. Toxicol., 36, 879-896.

xiv. Tareke, E. and Rydberg, P. (2002). "Analysis of acrylamide, a carcinogen formed in heated foodstuffs". J. Agric. Food. Chem.50 (17): 4998-5006.

xv. Vesper, H. W.; Bernert, J. T.; Ospina, M.; Meyers, T.; Ingham, L.; Smith, A.; Myers, G. L. (2007). "Assessment of the Relation between Biomarkers for Smoking and Biomarkers for Acrylamide Exposure in Humans".Cancer Epidemiology Biomarkers \& Prevention16 (11): 2471.

xvi. Zyzak, D. V., Sanders, R. A., Stojanovic, M., Tallmadge, D. H., Eberhart, B. L., Ewald, D. K., ... and Villagran, M. D. (2003). Acrylamide formation mechanism in heated foods. Journal of agricultural and food chemistry, 51(16), $4782-4787$.

xvii. Grob, K., Biedermann, M., Biedermann-Brem, S., Noti, A., Imhof, D., Amrein, T. (2003). French chips with less than $100 \mathrm{mg} / \mathrm{kg}$ acrylamide. A collaboration between cooks and analysts. European Food Research and Technology, 217, 185-194.

xviii. Olsson, K., Svensson, R., and Roslund, C.-A. (2004). Tuber components affecting acrylamide formation and colour in fried potato: variation by variety, year, storage temperature and storage time. Journal of the Science of Food Agriculture, 84, 447-458.

Williams, J. S. E. (2005). Influence of variety and processing conditions on acrylamide levels in fried potato crisps. Food Chemistry, 90, 875-881.

xix. Matthäus, B., Haase, N. U., and Vosmann, K. (2004). Factors affecting the concentration of acrylamide during deep-fat frying of potatoes. European Journal of Lipid Science and Technology, 106(11), 793-801.

xx. Ogolla, J. A. (2013). Acrylamide Contamination in Commercial Potato Crisps in Kenya: Levels of Intake and Effects of Processing Parameters in Local Cultivars (Doctoral dissertation, University of Nairobi).

xxi. Pedreschi, F., Kaack, K., and Granby, K. (2004). Reduction of acrylamide formation in potato slices during frying. LWT-Food Science and Technology, 37(6), 679-685.

xxii. Pedreschi, F., Moyano, P., Kaack, K., and Granby, K. (2005). Colour changes and acrylamide formation in fried potato slices. Food Research International, 38(1), 1-9. 
xxiii. Vural Go "kmen, Tunc Koray Palazog and Hamide Z. Senyuva, (2007). Relation between the acrylamide formation and time-temperature history of surface and core regions of French chips.

xxiv. Mottram, D.S., Wedzicha, B.L., and Dodson, A.T. (2002). Food chemistry: acrylamide is formed in the Maillard reaction. Nature, 419(6906), 448-449.

xxv. Stadler, R.H., Blank, I., Varga, N., Robert, F., Hau, J., Guy, P.A., and Riediker, S. (2002). Food chemistry: acrylamide from Maillard reaction products. Nature, 419(6906), 449-450.

xxvi. El-Ghorab, A.H., Fujioka, K. and Shibamoto, T. (2006). Determination of acrylamide formed in asparagine Dglucose maillard model systems by using gas chromatography with headspace solid-phase micro extraction. J. AOAC Int., 89(1): 149-153.

xxvii. Zhang Yu, Ren, Y, Zhang Y (2009). New research developments on acrylamide: analytical chemistry, formation mechanism, and mitigation recipes. Chem. Rev., 109(9): 4375-4397. 